\title{
Honey Inhibitory Effect on Cancer
}

\section{ISSN: 2640-9208}

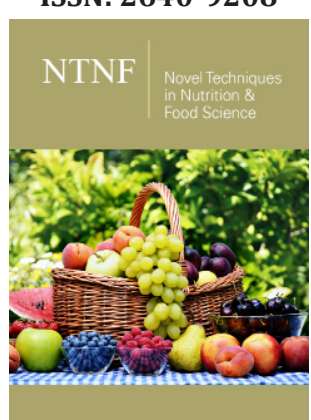

${ }^{* 1}$ Corresponding author: Pinheiro L, iMed.UL-FFUL, Lisbon, Portugal

Submission: 眥July 02, 2019

Published: 制July 09, 2019

Volume 4 - Issue 1

How to cite this article: Pinheiro $\mathrm{L}$ Florack A, Faustino C. Honey Inhibitory Effect on Cancer. Nov Tech Nutri Food Sci. 4(1).NTNF.000580.2019.

DOI: 10.31031/NTNF.2019.04.000580

Copyright@ Pinheiro L, This article is distributed under the terms of the Creative Commons Attribution 4.0 International License, which permits unrestricted use and redistribution provided that the original author and source are credited.

\author{
Pinheiro $\mathrm{L}^{1 *}$, Florack $\mathrm{A}^{2}$ and Faustino $\mathrm{C}^{1}$ \\ iMed.UL-FFUL, Lisbon, Portugal \\ ${ }^{2}$ University of Kansas School of Pharmacy, USA
}

\begin{abstract}
Cancer is one of the most common causes of death worldwide. Current treatments for cancer include chemotherapy, radiotherapy and surgery. One significant setback to cancer treatment is the lack of specificity. Chemotherapeutics are not specific for only cancer cells and as a result side effects are common. Due to this, there is a focus in research now on new and improved cancer treatments. Natural products are a focus point for this research. Honey is a natural product produced by honeybees with known anticancer effects [1-5]. Much research today is focused on honey's constituents and potential medicinal properties. This mini review will discuss the research to date on honey and explain some mechanisms of honey's anticancer properties.
\end{abstract}

Keywords: Honey; Cancer inhibition; Antitumoral; Antiproliferative; Phenolic; Flavonoid

\section{Introduction}

Bees naturally produce honey from the nectar of flowers. It is a supersaturated solution of over 200 compounds, predominantly consisting of sugars (75\% fructose and glucose). Other ingredients include phenolic compounds (flavonoids, phenolic acids), proteins, minerals, organic acids, water, vitamins (B complex), enzymes, volatile compounds, and pigments [16]. Throughout history honey has been used as a natural supplement, as both a food and medicine. Clinical trials and in vitro studies have proven to show that honey has several medicinal properties such as antimicrobial, antioxidant, anti-inflammatory, antitumor, and wound healing abilities [3]. Over the years there have been multiple studies involving honey and its pharmacological and biological effects. Honey has attracted the attention of researchers as a complementary and alternative treatment in contemporary medicine in the field of oncology [2]. The World Health Organization (WHO) estimates that by 2020 there will be more than 15 million new cases of cancer [5]. One significant problem in the field of oncology is that despite considerable research, cancer still poses as a challenge to prevent and treat due to unfortunate and often serious side effects, which has led to the search of novel, more tolerable treatments. The search has dabbled in natural products and food that may have chemoprotective and chemotherapeutic properties [3]. A natural product that has drawn attention for having these properties is honey. In the last decade multiple studies have been conducted looking specifically at honey and its antitumor and antiproliferative properties. Laboratory tests and clinical trials have tried to pinpoint honey's mechanisms of action for these abilities. This study is a review of current research findings of the use of honey as an anticancer agent.

Flavonoids and phenolic compounds in honey are what give honey its antitumor and anticancer properties $[2,4,5]$. Its biologic activity is dependent on many factors including honeybee species, nectar source, geographical region, climate and how the honey is processed [5]. This is one reason that makes research on honey challenging. With different compositions and honey sources, it is hard to standardize what honey is truly capable of and get continued replicable results. There have been around 300 types of honey recognized, with gelam, Manuka, tualang, buckwheat, acacia, Greek thyme, pine and fir honeys common types found in research studies. Honey's anticancer properties have been tested in breast, colorectal, prostate, oral, cervical, endometrial, and renal cancer cell lines [5], using animal models and measuring tumor growth, size number, and position, for example. Tualang and Manuka honeys have been investigated for their potential anticancer abilities [2]. Several processes 
have been identified, such as: cell cycle arrest, mitochondrial pathway activation (including induction of the mitochondrial outer membrane permeabilization), induction of apoptosis, modulation of oxidative stress, inhibition of inflammation, and modulating estrogenic activity, to name only a few. The cell cycle is a tightly controlled process of cell growth and proliferation involving four successive stages: M, G1, G2, and S.

This entire process is under direction of a cascade of protein kinases and checkpoints. In healthy cells these events are controlled but in cancer cells the cell cycle becomes unregulated and can cause uninhibited cell proliferation. Experimental data show that several phenolic compounds and flavonoids (chrysin, quercetin, and kaempferol) in honey have the ability to cause cell cycle arrest at the G1 phase $[4,5]$. Two available methods used to cure cancer, chemotherapy and radiotherapy, cause cell death by activating the mitochondrial pathway, also called the intrinsic pathway. Honeyspecifically flavonoids-can activate the mitochondrial pathway and release proteins like cytochrome-C, causing cell death. This is a common mechanism for agents with anticancer abilities [4,5]. Apoptosis is programed cell death. Irregular apoptotic turnover and uncontrolled cell growth are two prominent characteristics of cancer cells.

A study comparing gelam honey, 5-fluorouracil (5-FU), and their combination was conducted in vitro on colorectal cancer cells (HT-29) to determine the apoptotic and cytotoxic effects of gelam honey alone and in combination with 5-FU on HT-29 cells [3]. Results showed a significant increase in the number of apoptotic cells after treatment with gelam honey, indicating that honey can induce apoptotic pathways. More specifically honey is shown to cause apoptotic cell death through inducing the activation of the caspase cascade [4], promoting the expression of proapoptotic proteins, such as Bax, and inhibiting the expression of antiapoptotic proteins such as Bcl-2. Honey also modulates p53 expression (a tumor suppressor) and DNA fragmentation. Honey's pro-apoptosis effects have been shown to be enhanced when combined with other natural product such as Aloe Vera [1]. Honey is well known for being an antioxidant and free radical scavenger, activities accounted for by its flavonoids and phenolic compounds. It also can create hydrogen peroxide, $\mathrm{H}_{2} \mathrm{O}_{2}$. Honey can act like a double-edged sword. It can behave like a pro-oxidant and create more ROS to increase oxidative stress and it can behave as an antioxidant and eat up free radicals [4]. Some honey types (Tualang and Manuka) have been shown to decrease inflammation in cell cultures and animal models. Studies in rats with breast cancer indicated a decrease in monocyte levels (which produce cytokines) and TNF- $\alpha$ levels in blood [2]. Honey has also been shown to have the ability to decrease the activities of inflammatory mediators' cyclooxygenase-1 (COX1) and cyclooxygenase -2 (COX-2) [6]. The phenolic compounds in honey suppress proinflammatory action of inducible nitric oxide synthase (iNOS) and COX-2 [5]. Breast cancer is the most common type of cancer in women. Many breast cancers are related to estrogen activity, specifically the levels of endogenous estrogen (E2) and estrogen receptors. Phenolic compounds are phytoestrogens - plant-derived, natural compounds that are structurally like mammalian estrogen which can bind to estrogen receptors in the body. As a result of this, honey has been shown to have a biphasic action inside the body depending on its concentration. At low concentrations honey was seen to have antiestrogenic activity while at high concentrations it has stimulatory effects [4].

\section{Conclusion}

While there is still debate about the precise mechanisms, researchers have shown that honey has anticancer effects. A challenge to honey research is the vast varieties of honeys. Honey can be found all over the world, but no two types are the same. They have different constituent concentrations which results in varied biological and pharmaceutical properties. Today there is no standardization of the type of honey used in research. More studies are needed to authenticate the true capabilities of natural honey, both in tissue cultures and animal models, including humans, as well as the use of honey alone and as an adjuvant. Studies have also shown that honey has synergistic effects with current chemotherapeutics. This merits additional investigations into the use of honey as an adjuvant and how precisely it is synergistic with chemotherapeutics. The evidence that proves honey has anticancer properties is out there; now research needs to focus on replicating these results consistently so that more clinical trials can be conducted in humans.

\section{References}

1. Porcza LM, Simms C, Chopra M (2017) Honey and cancer: current status and future directions. diseases 4(4): 30.

2. Ahmed S, Sulaiman SA, Othman NH (2017) Oral administration of tualang and manuka honeys modulates breast cancer progression in sprague-dawley rats model. Evid Based Complement Alternat Med.

3. Johari SAT, Hashim F, Ismail WI, Ali AM (2019) Combinatorial cytotoxic effects of gelam honey and 5-fluorouracil against human adenocarcinoma colon cancer ht-29 cells in vitro. International Journal of Cell Biology, P.10.

4. Erejuwa 00, Sulaiman SA, Wahab MS (2014) Effects of honey and its mechanisms of action on the development and progression of cancer. Molecules 19(2): 2497-2522.

5. Waheed M, Hussain MB, Javed A, Mushtaq Z, Hassan S. et al. (2018) Honey and cancer: A mechanistic review. Clinical Nutrition.

6. Faustino C, Pinheiro L (2015) Antimicrobial properties and therapeutic benefits of honey in the quest for more efficient antimicrobial agents. In: Vilas MA (Ed.), The battle against microbial pathogens: basic science, technological advances and educational programs. Formatex Research Center, Badajoz, Spain. 